\title{
Epidemic Spreading in a Social Network with Facial Masks wearing Individuals
}

\author{
Duan-Shin Lee, Senior Member, IEEE, and Miao Zhu
}

\begin{abstract}
In this paper, we present a susceptible-infectedrecovered (SIR) model with individuals wearing facial masks and individuals who do not. The disease transmission rates, the recovering rates and the fraction of individuals who wear masks are all time dependent in the model. We develop a progressive estimation of the disease transmission rates and the recovering rates based on the COVID-19 data published by John Hopkins University. We determine the fraction of individual who wear masks by a maximum likelihood estimation, which maximizes the transition probability of a stochastic susceptibleinfected-recovered model. The transition probability is numerically difficult to compute if the number of infected individuals is large. We develop an approximation for the transition probability based on central limit theorem and mean field approximation. We show through numerical study that our approximation works well. We develop a bond percolation analysis to predict the eventual fraction of population who are infected, assuming that parameters of the SIR model do not change anymore.

We predict the outcome of COVID-19 pandemic using our theory.
\end{abstract}

keywords:bond percolation; epidemic network; susceptibleinfected-recovered model; masks; COVID-19

\section{INTRODUCTION}

In December of 2019 a few patients of a new infectious respiratory disease were detected in Wuhan, China. This disease has been called coronavirus disease 2019 (COVID-19) and the virus that causes COVID-19 has been named SARS-CoV-2 by the World Health Organization (WHO). WHO declared the outbreak a public health emergency of international concern at the end of January 2020, and a pandemic on March 11, 2020. Since the outbreak, most countries have adopted various measures in an attempt to contain the pandemic. These measures include restriction of travelling, shutting down schools, restaurants and businesses, cancelling large gatherings such as concerts, sports and religious activities, and even city lockdowns where residents are not allowed to leave home unless emergencies. Clearly these measures seriously affect daily lives and are devastating to the economics. The purpose of this paper is to show that wearing facial masks is a simple and inexpensive measure to contain the spread of COVID-19. In fact, we shall show that if a relatively small fraction of population wears facial masks, the disease can be contained.

The authors are with the Institute of Communications Engineering, National Tsing Hua University, Hsinchu 30013, Taiwan, R.O.C. Email: lds@cs.nthu.edu.tw, xmzhumiao11111@hotmail.com. This research was supported in part by the Ministry of Science and Technology, Taiwan, R.O.C., under Contract 109-2221-E-007-093-MY2.
Facial masks have been shown in labs to be effective to limit the spread of droplets or aerosols if a wearer coughs [1][4]. This ability is measured by a quantity called the outward mask filter efficiency of masks. Facial masks also protect their wearers from inhaling droplets or aerosols from a nearby cougher, if the cougher does not wear a mask. This ability is measured by a quantity called the inward mask filter efficiency of masks. Thus, facial masks can be particularly useful to confine the spreading of diseases that transmit through droplets or aerosols. However, the effect of wearing facial masks to the epidemic spreading has never been studied in a network level. In this paper we present an epidemic network study to justify this argument. Specifically, we propose a time dependent susceptible-infected-recovered (SIR) model with two types of individuals. Type 1 individuals wear a facial mask and type 2 individuals do not. A randomly selected individual from a population is a type 1 individual with probability $p$, and is of type 2 with probability $1-p$. There are four types of contacts between two individuals depending on whether the two individuals wear a facial mask or not. These four types of contacts have four different disease transmission rates. From the data published by John Hopkins University [5] we progressively estimate the time dependent disease transmission rates and the recovery rates of the SIR model.

For parameter $p$, we propose a stochastic version of the SIR model. Specifically, we assume that the COVID-19 epidemic propagates in a social contact network according to an independent cascade model [6], [7]. The social contact network in our study consists of tree connection of cliques of random sizes. We derive the transition probability of the number of infected individuals from one time slot to the next. We propose a maximum likelihood estimation of $p$ that maximizes the transition probability. The transition probability is expressed in terms of binomial distributions. Parameters of the binomial distributions corresponding to real data published in [5] are typically very large. That makes the transition probability numerically difficult to compute. We propose an approximation of the transition probability based on central limit theorem and mean field approximation. Through numerical studies, we show that the approximation works well. We derive a percolation analysis of the maximum number of individuals that can eventually be infected. We incorporate the maximum likelihood estimation and the percolation analysis into the progressive estimation. That is, based on the data published by John Hopkins University, we progressively estimate the disease transmission rates and the recovery rates. We then find $p$ from the maximum likelihood estimation. Finally, using 
percolation analysis, we predict the maximum number of individuals that can eventually be infected.

COVID-19 has attracted a lot of research work on epidemic networks. We now review some research work that is related to the use of facial masks. Li et al. [8] proposed a decision making process in a susceptible-exposed-symptomaticquarantined model. At each time, every susceptible individual chooses whether to wear a mask or not in the next time step, which depends on an evaluation of the potential costs and perceived risk of infection. Damette [9] conducted a panel econometric exercise to assess the dynamic impact of face mask use on both infected cases and fatalities at a global scale. A negative impact of mask wearing on fatality rates and on the COVID-19 number of infected cases was observed. Damette found that population density and pollution levels are significant determinants of heterogeneity regarding mask adoption across countries, while altruism, trust in government and demographics are not. In addition, the most effective way to increase mask use is through strict laws by governments. Betsch [10] examined nearly 7000 German participants and observed that implementing a mandatory policy increased actual compliance of wearing masks. Mask wearing correlated positively with other protective behaviours. They also observed that voluntary policy is far less effective, and mandatory policy is far more effective to curb transmission of airborne virus.

The outline of this paper is as follows. In Section III we present a time dependent SIR model. We present a progressive estimation of the disease transmission rates and the recovery rates of the model. In Section III. we present a social contact network consisting of tree connection of cliques. We present a maximum likelihood method to estimate $p$. In Section III-A. we derive the clustering coefficient of this social contact network. In Section IV] we present a percolation analysis. In Section $\mathrm{V}$ we present results of numerical study and simulation. We present the conclusions in Section VI

\section{TIMe Dependent SIR ModeL}

In this section we present a discrete-time susceptibleinfected-recovered (SIR) model. SIR models have long been used to model and study epidemics [11]. We now survey a few recent research studies that are related to SIR models. Opuszko et al. [12] studied the impact of the network structure on the SIR model spreading phenomena. This study is based on simulation of SIR models on real life online social networks as well as mathematical network models. Bernardes et al. [13] studied SIR models on P2P systems. The study was also simulation based. Wang et al. [14] and Zheng et al. [15] used two-layer multiplex networks to investigate the multiple influence between awareness diffusion and epidemic propagation, where the upper layer represents the awareness diffusion regarding epidemics and the lower layer expresses the epidemic propagation. Wang et al. [14] analytically showed that the epidemic threshold is correlated with the awareness diffusion as well as the topology of epidemic networks.

In our model time is divided into periods of equal lengths. There are two types of individuals. Type 1 individuals wear a facial mask and type 2 individuals do not. A randomly selected individual from a population is a type 1 individual with probability $p(t)$ in period $t$. A randomly selected individual is of type 2 with probability $1-p(t)$. Let $s(t)$ and $r(t)$ be the number of susceptible and recovered individuals, respectively, at time $t$. Similarly, let $x_{i}(t)$ be the number of infected type $i$ individuals in period $t$ for $i=1,2$. In this model, the disease transmission rates are time dependent. Let $\beta_{i j}(t)$ be the expected number of type $j$ susceptible individuals who receive the disease from one type $i$ infected individual per unit time in period $t$. Let $\gamma(t)$ be the recovering rate of the disease in period $t$. We assume that both types of infected individuals have the same recovering rate.

In this paper we assume that the length of a time unit in the discrete-time SIR model is $\tau$ days. The dynamics of this discrete-time SIR model is as follows. The existing infected individuals at time $t$ - transmit the disease to newly infected individuals. Those infected individuals existed at time $t-$ become recovered at time $(t+1)-$. It is easy to derive the following set of difference equations for the SIR model.

$$
\begin{aligned}
& s(t+1)-s(t) \\
& =-\left(\sum_{i=1}^{2} x_{i}(t)\left[\beta_{i, 1}(t) p(t)+\beta_{i, 2}(t)(1-p(t))\right]\right) \frac{s(t)}{n} \\
& x_{1}(t+1)-x_{1}(t)=x_{1}(t) \beta_{11}(t) \frac{s(t) p(t)}{n} \\
& \quad+x_{2}(t) \beta_{21}(t) \frac{s(t) p(t)}{n}-\gamma(t) x_{1}(t) \\
& x_{2}(t+1)-x_{2}(t)=x_{1}(t) \beta_{12}(t) \frac{s(t)(1-p(t))}{n} \\
& \quad+x_{2}(t) \beta_{22}(t) \frac{s(t)(1-p(t))}{n}-\gamma(t) x_{2}(t) \\
& r(t+1)-r(t)=\gamma(t)\left(x_{1}(t)+x_{2}(t)\right)
\end{aligned}
$$

where

$$
n=s(t)+x_{1}(t)+x_{2}(t)+r(t)
$$

is the size of the population. We assume that the epidemics is in the early stage. That is, we assume that $s(t) \approx n$. Under this assumption, the difference equations for $x_{1}(t)$ and $x_{2}(t)$ reduce to

$$
\begin{aligned}
x_{1}(t+1)-x_{1}(t)= & \left(x_{1}(t) \beta_{11}(t)+x_{2}(t) \beta_{21}(t)\right) p(t) \\
& -\gamma(t) x_{1}(t) \\
x_{2}(t+1)-x_{2}(t)= & \left(x_{1}(t) \beta_{12}(t)+x_{2}(t) \beta_{22}(t)\right)(1-p(t)) \\
& -\gamma(t) x_{2}(t) .
\end{aligned}
$$

We now determine parameters $\beta_{i j}(t)$ and $p(t)$. We reduce the number of parameters. Previous study [2] suggested that

$$
\begin{aligned}
& \beta_{12}(t)=\left(1-\eta_{1}\right) \beta_{22}(t) \\
& \beta_{21}(t)=\left(1-\eta_{2}\right) \beta_{22}(t),
\end{aligned}
$$

where $\eta_{1}$ and $\eta_{2}$ are outward and inward efficiencies of masks, respectively. Study [4] suggested that $\eta_{1}>\eta_{2}$. In addition, we assume that

$$
\beta_{11}(t)=\left(1-\eta_{1}\right)\left(1-\eta_{2}\right) \beta_{22}(t)
$$


Thus, we only need to determine $\beta_{22}(t)$. The other three parameters are determined according to Eqs. (4), (5) and (6). Substituting (4), (5) and (6) into (2) and (3), we obtain

$$
\begin{aligned}
x_{1}(t+1)= & \beta_{22}(t)\left[x_{1}(t)\left(1-\eta_{1}\right)\left(1-\eta_{2}\right)\right. \\
& \left.+x_{2}(t)\left(1-\eta_{2}\right)\right] p(t)+(1-\gamma(t)) x_{1}(t) \\
x_{2}(t+1)= & \beta_{22}(t)\left[x_{1}(t)\left(1-\eta_{1}\right)+x_{2}(t)\right](1-p(t)) \\
& +(1-\gamma(t)) x_{2}(t) .
\end{aligned}
$$

Now we determine the values of parameters $\gamma(t), \beta_{22}(t)$ and $p(t)$ based on the data published by John Hopkins University [5]. Note that John Hopkins University publishes total number of daily newly infected individuals and the number of recovered individuals. From the published data, one can easily compute $r(t)$ for each $t$. The website does not distinguish between infected individuals who wear masks or who do not. Thus, we must determine $\beta_{22}(t)$ and $p(t)$ based on the total number of infected individuals

$$
x(t)=x_{1}(t)+x_{2}(t)
$$

at time $t$. From the data published by John Hopkins University, we estimate $\gamma(t), \beta_{22}(t)$ and $p(t)$. We develop in section $[\mathrm{IV}$ a percolation analysis to determine the ultimate size of infected population, if these parameters do not change.

Recovering rate is simple to determine. From (1) we have

$$
\gamma(t)=\frac{r(t+1)-r(t)}{x(t)} .
$$

Next, we determine $\beta_{22}(t)$. To determine the value of $\beta_{22}(t)$ in period $t$, we let $x(t)$ be the total number of infected individuals in period $t$, i.e.

Adding Eqs. (7) and (8) and solving for $\beta_{22}(t)$, we obtain (10) displayed in Fig. 1 .

To determine the value of $\beta_{22}(t)$ at time $t$, we use $x(t+1)$ published in [5]. We assume that $x_{1}(t)$ and $x_{2}(t)$ and $p(t)$ are available for $t$. We use 10 to determine $\beta_{22}(t)$. The value of $p(t)$ is determined by a maximum likelihood estimation method. We shall present this method in Section III After $\beta_{22}(t)$ at time $t$ is determined, we use (7) and (8) to determine $x_{1}(t+1)$ and $x_{2}(t+1)$. Note that the sum of $x_{1}(t+1)$ and $x_{2}(t+1)$ determined in this way agrees with $x(t+1)$.

We summarize the algorithm that determines parameters of the time-dependent SIR model in Algorithm 1 .

\section{Maximum Likelihood Estimation of $p(t)$}

In this section we present a maximum likelihood estimation method to determine the value $p(t)$ based on $x(t), x(t+1)$ and $\beta_{22}(t-1)$. This estimation will be progressively used by Algorithm 1 in periods $t=2,3, \ldots$. Thus, in the rest of this paper we simplify the notation by eliminating the dependency of time from $p(t)$ and $\gamma(t)$ and simply use $p$ and $\gamma$, respectively.

Recall that $x(t)$ and $x(t+1)$ are the total number of infected individuals in periods $t$ and $t+1$. We would like to determine the value of $p$ such that the likelihood of this sample path is maximized. To determine the likelihood function, we propose

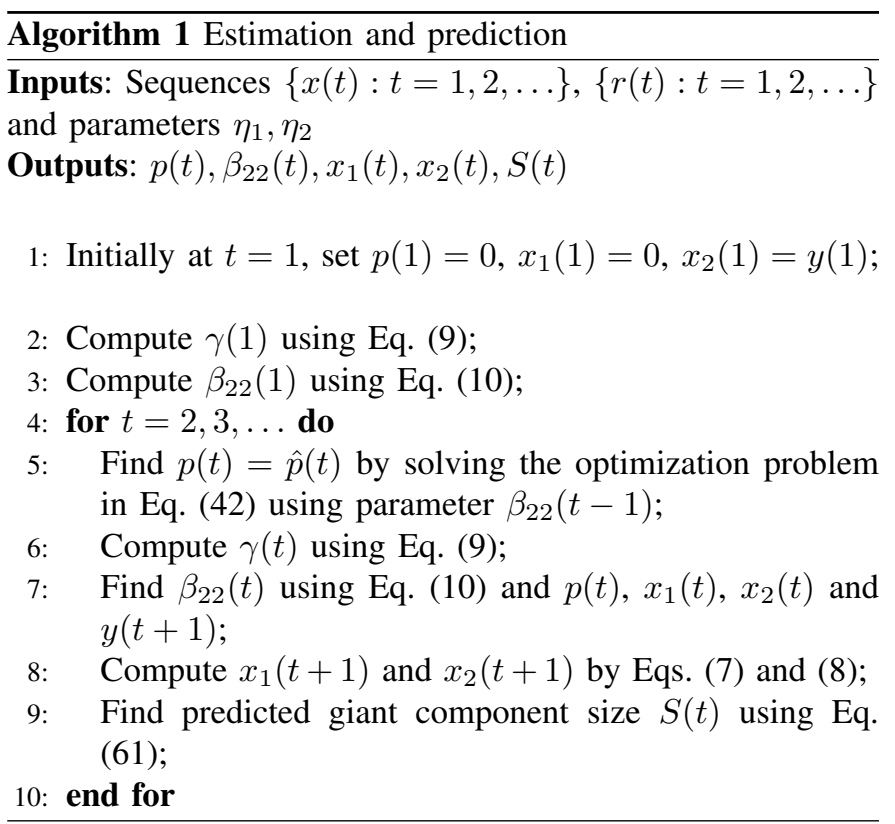

a social network model and a probabilistic version of the SIR model to spread the disease in this network. We first describe the social network model and then the probabilistic SIR model. It is well known that social networks possess a few important properties, such as significant clustering coefficients, power law degree distributions, short path lengths, positive degreedegree correlations, existence of community structures and etc. In this paper, we propose a social network model that possesses significant clustering coefficients and allow analysis of epidemic spreading at the same time. Our social network model is a tree connection of cliques of random sizes arranged in layers. Specifically, in layer zero, there is a clique consisting of a random number of fully connected vertices. Each vertex in a layer connects to $k$ cliques of random sizes in the layer. We model dense clusters or households in social networks by cliques. Randomly connected cliques have been proposed to model social networks by Coupechoux et al. [16] and Trapman [17]. We illustrate a graphical example of this model in Figure 2 Let $K$ be the size of a generic clique. Assume that the probability mass function (pmf) of $K$ is given, i.e.

$$
F(i)=\mathrm{P}(K=i), \quad i=1,2, \ldots
$$

From this pmf, we compute recursively the $n$-th convolution with itself, i.e.

$$
F^{(n)}(i)=\sum_{j=0}^{i} F^{(n-1)}(i-j) F(j) .
$$

Let $k_{c}$ and $\sigma_{c}^{2}$ be the mean and variance of $K$, i.e.

$$
\begin{aligned}
& \mathrm{E}[K]=k_{c} \\
& \operatorname{Var}(K)=\sigma_{c}^{2} .
\end{aligned}
$$

This model clearly has a non-vanishing clustering coefficient. We derive the clustering coefficient of this model at the end of this section. 


$$
\beta_{22}(t)=\frac{x(t+1)-(1-\gamma(t)) x(t)}{x_{1}(t)\left[\left(1-\eta_{1}\right)\left(1-\eta_{2}\right) p(t)+\left(1-\eta_{1}\right)(1-p(t))\right]+x_{2}(t)\left[\left(1-\eta_{2}\right) p(t)+(1-p(t))\right]} .
$$

Fig. 1. An expression of $\beta_{22}(t)$.

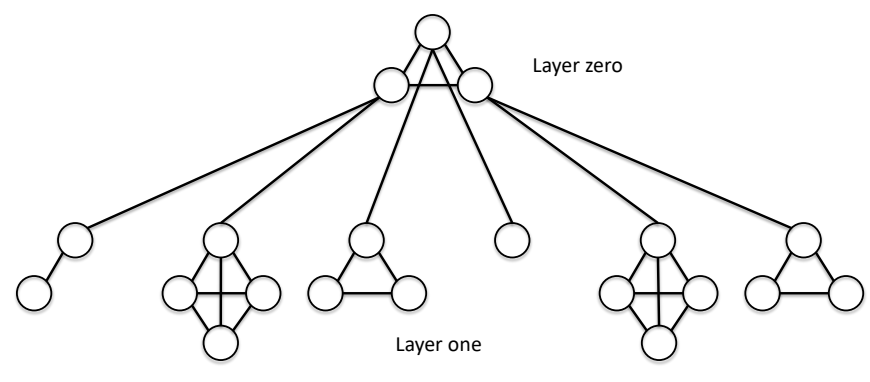

Fig. 2. An illustration of the social network, in which an epidemic spreads. In this example, $k=2$. The size of cliques is random.

To determine the likelihood function, we propose a probabilistic version of the SIR model that spreads the disease in the social network model described in the last paragraph. This probabilistic SIR model is based on independent cascade models. Independent cascade models are popular not only in the study of epidemic spreading but also in the influence maximization problems of viral marketing [6], [7]. In an independent cascade model, each infected node has exactly one opportunity to transmit the disease to its neighbors. Whether the transmissions are successful or not depend on independent events. In our model, we distinguish between nodes that have used their opportunity to transmit the disease from those who have not. A node is said to be infectious, if this node has not used its opportunity to transmit the disease to its neighbors. A node is said to be infected but not infectious, if it has used its opportunity to transmit the disease. Our model is a discrete time model. Let $X_{t}$ be the total number of currently infected individuals in period $t$. Let $Y_{t}$ be the number of individual who contract the disease in period $t$. In our model, we assume that a layer $i$ vertex who contracts the disease in period $t$ have ability to transmit the disease to their neighbors in layer $i+1$ in period $t+1$, and lose the ability in periods $t+2, t+3, \ldots$ and so on. Recall that we use cliques to model dense clustering such as households in a social network, Since householdbased transmission is typically much stronger than that outside households [18], [19], we assume that once a vertex in a clique becomes infected, all vertices in that clique are infected at once. In period $t$, there are $Y_{t}$ infectious individuals and $X_{t}-Y_{t}$ infected individuals who can not transmit the disease to others. Those who are infected but cannot transmit the disease to others in period $t$ can remain infected in subsequent periods or become recovered with probability $\gamma$. A graphical illustration of the model is shown in Figure 3 It is clear that this model is a discrete time Markov chain. We shall derive the transition probability

$$
\mathrm{P}\left(X_{t+1}=x(t+1), Y_{t+1}=y(t+1) \mid X_{t}=x(t), Y_{t}=y(t)\right)
$$

and find $\hat{p}(t)$ such that

$$
\begin{gathered}
\hat{p}(t)=\underset{0 \leq p \leq 1}{\operatorname{argmax}} \log \left(\mathrm { P } \left(X_{t+1}=x(t+1), Y_{t+1}=y(t+1) \mid\right.\right. \\
\left.\left.X_{t}=x(t), Y_{t}=y(t)\right)\right) .
\end{gathered}
$$

We remark that our assumption that infected individuals can transmit the disease in the beginning of their infection periods, and lose the ability to transmit in the later part of the infection periods has nothing to do the real dynamics of the disease. In independent cascade models each infected vertex has exactly one opportunity to transmit the disease to its neighbors. An infected vertex can execute its opportunity in any one time slot during its infection period. In this paper, we assume that infected vertices execute their opportunities in the beginning of infection periods. We make this assumption so that the mathematical analysis of 13 is possible. In this paper we have not attempted to model the microscopic transmission dynamics of COVID-19. In fact, [20], [21] showed that COVID-19 patients typically show symptoms before they can transmit the disease.

We now specify more details of the probabilistic SIR model. Each individual can be of type 1, or of type 2. Each infectious individual has $k$ contacts, to whom he or she can transmit the disease. A type $i$ infectious individual can transmit the disease to a type $j$ susceptible individual with probability $\phi_{i j}$, where $i, j=1,2$. Parameter $\phi_{i j}$ is related to $\beta_{22}(t)$ and $\gamma$ through

$$
\phi_{i j}=\frac{\beta_{i j}(t) / \gamma}{k} \text {. }
$$

Let $C$ be the number of type 1 individuals among the $Y_{t}$ infectious individuals in period $t$. Conditioning on event $\{C=i\}$, one can express $Y_{t+1}$ in terms of $Y_{t}$ in the following manner. Let

$$
\begin{aligned}
& I_{1}=\sum_{j=1}^{i k} 1_{\left\{U_{j}=1\right\}} \\
& I_{2}=\sum_{j=1}^{\left(Y_{t}-i\right) k} 1_{\left\{V_{j}=1\right\}} .
\end{aligned}
$$

$\left\{U_{j}, j=1,2, \ldots\right\}$ and $\left\{V_{j}, j=1,2, \ldots\right\}$ are two independent and identically distributed (i.i.d.) sequences of Bernoulli 
random variables with success probabilities $p_{1}$ and $p_{2}$, respectively. The two sequences are independent to anything else. Event $\left\{U_{j}=1\right\}$ (resp. event $\left\{V_{j}=1\right\}$ ) indicates a type 1 (resp. type 2) infectious individual successfully transmits the disease to a neighboring node in the next layer. Thus, $I_{1}$ (resp. $I_{2}$ ) is the number of cliques that are infected by type 1 (resp. type 2) infectious individuals. Recall that we assume that all members in a clique are infected, if one member in the clique is infected. Individuals in these cliques are all infected and are infectious at time $t+1$. Thus, we can express $Y_{t+1}$ in terms of $I_{1}$ and $I_{2}$, i.e.

$$
Y_{t+1}=\sum_{j=1}^{I_{1}+I_{2}} K_{j}
$$

where we recall that $\left\{K_{j}\right\}$ is an i.i.d. sequence of random variables that denote random clique sizes. An infected individual becomes recovered with probability $\gamma$, and remains infected otherwise. Hence,

$$
X_{t+1}=Y_{t+1}+\sum_{j=1}^{X_{t}} 1_{\left\{W_{j}=1\right\}}
$$

where $\left\{W_{j}, j=1,2, \ldots\right\}$ is an i.i.d. sequence of Bernoulli random variable independent of anything else. The success probability of $W_{j}$ is $1-\gamma$. Let

$$
I_{3}=\sum_{j=1}^{X_{t}} 1_{\left\{W_{j}=1\right\}},
$$

and we rewrite $(19)$ as

$$
X_{t+1}=Y_{t+1}+I_{3} .
$$

We present a schematic description in Figure 3 .

We now analyze $p_{1}$ and $p_{2}$, which are the success probabilities of the Bernoulli random variables $U_{j}$ and $V_{j}$, respectively. Event $\left\{U_{j}=1\right\}$ indicates a type 1 infectious individual successfully transmits the disease to a neighboring node. This occurs with probability $p_{1}$, where

$$
p_{1}=\phi_{11} p+\phi_{12}(1-p)
$$

Similarly, event $\left\{V_{j}=1\right\}$ indicates a type 2 infectious individual successfully transmits the disease to a neighboring node with probability $p_{2}$, where

$$
p_{2}=\phi_{21} p+\phi_{22}(1-p) \text {. }
$$

Conditioning of event $\{C=i\}$ and using $(18)$ and $(19)$, we have

$$
\begin{aligned}
& \mathrm{P}\left(X_{t+1}=x(t+1), Y_{t+1}=y(t+1) \mid X_{t}=x(t)\right. \\
& \left.\quad Y_{t}=y(t), C=i\right)= \\
& \mathrm{P}\left(I_{3}=x(t+1)-y(t), \sum_{j=1}^{I_{1}+I_{2}} K_{j}=y(t+1) \mid X_{t}=x(t)\right. \\
& \left.\quad Y_{t}=y(t), C=i\right)
\end{aligned}
$$

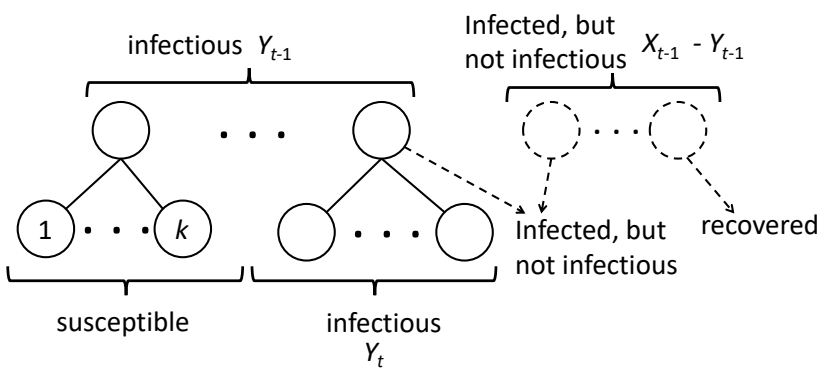

Fig. 3. A probabilistic SIR model. Nodes shown in solid line are infectious. Nodes shown in dashed line are infected, but not infectious. Each infectious root node has exactly $k$ contact cliques. Infected nodes that are not infectious can remain infected in the next period, or become recovered.

Conditioning on event $\left\{C=i, I_{1}=i_{1}, I_{2}=i_{2}, I_{3}=j\right\}$, the transition probability is

$$
\begin{aligned}
& \mathrm{P}\left(X_{t+1}=x(t+1), Y_{t+1}=y(t+1) \mid X_{t}=x(t),\right. \\
& \left.\quad Y_{t}=y(t), C=i, I_{1}=i_{1}, I_{2}=i_{2}, I_{3}=j\right) \\
& =F^{\left(i_{1}+i_{2}\right)}(y(t+1)),
\end{aligned}
$$

where $F^{(n)}$ is the $n$-th convolution of the clique size pmf with itself. Conditioning on $\{C=i\}$, random variables $I_{1}, I_{2}$ and $I_{3}$ all have binomial distributions. Define binomial probability mass function

$$
b(x, n, r) \stackrel{\text { def }}{=} \begin{cases}\left(\begin{array}{c}
n \\
x
\end{array}\right) r^{x}(1-r)^{n-x} & x=0, \ldots, n \\
0 & \text { otherwise. }\end{cases}
$$

The conditional distributions of $I_{1}, I_{2}$ and $I_{3}$ are

$$
\begin{aligned}
& \mathrm{P}\left(I_{1}=j \mid X_{t}=x(t), Y_{t}=y(t), C=i\right)=b\left(j, i k, p_{1}\right) \\
& \mathrm{P}\left(I_{2}=j \mid X_{t}=x(t), Y_{t}=y(t), C=i\right) \\
& \quad=b\left(j,(y(t)-i) k, p_{2}\right) \\
& \mathrm{P}\left(I_{3}=j \mid X_{t}=x(t), Y_{t}=y(t), C=i\right) \\
& \quad=b(j, x(t), 1-\gamma) .
\end{aligned}
$$

Note that $I_{1}, I_{2}$ and $I_{3}$ are independent. Unconditioning by taking average on (25) with respect to the pmfs of $I_{1}, I_{2}$ and $I_{3}$ in (26), 27) and (28), we obtain (29) displayed in Fig. 4 In addition,

$$
\mathrm{P}\left(C=i \mid Y_{t}=y(t)\right)=b(i, y(t), p) .
$$

Taking average on 25 with respect to event $\{C=i\}$ using (30), we have (31) displayed in Fig. 5. In a special case, in which $K$ is degenerate and is equal to $k_{c}$, i.e $\operatorname{Pr}\left(K=k_{c}\right)=1$, (31) leads to a slightly simpler expression as follows.

Both Eq. (31) and Eq. (32) are very complicated to evaluate and they are difficult to be directly used in the optimization problem (14) when $y(t)$ or $y(t+1)$ is large. We now propose an approximation method to simplify (31). Conditioning on 


$$
\begin{aligned}
& \mathrm{P}\left(X_{t+1}=x(t+1), Y_{t+1}=y(t+1) \mid X_{t}=x(t), Y_{t}=y(t), C=i\right)= \\
& \left(\sum_{i_{1}=0}^{i k} \sum_{i_{2}=0}^{(y(t)-i) k} F^{\left(i_{1}+i_{2}\right)}(y(t+1)) b\left(i_{1}, i k, p_{1}\right) b\left(i_{2},(y(t)-i) k, p_{2}\right)\right) b(x(t+1)-y(t), x(t), 1-\gamma)
\end{aligned}
$$

Fig. 4. Conditional transition probability. Variable $i$ denotes the number of type 1 infectious individuals. Variable $i_{1}$ (resp. $i_{2}$ ) denotes the number of newly infected individuals by type 1 (resp. type 2) infectious individuals.

$$
\begin{aligned}
& \mathrm{P}\left(X_{t+1}=x(t+1), Y_{t+1}=y(t+1) \mid X_{t}=x(t), Y_{t}=y(t)\right)= \\
& \sum_{i=0}^{y(t)} \sum_{i_{1}=0}^{i k} \sum_{i_{2}=0}^{(y(t)-i) k} F^{\left(i_{1}+i_{2}\right)}(y(t+1)) b\left(i_{1}, i k, p_{1}\right) b\left(i_{2},(y(t)-i) k, p_{2}\right) b(x(t+1)-y(t), x(t), 1-\gamma) b(i, y(t), p)
\end{aligned}
$$

Fig. 5. Transition probability. Variable $i$ denotes the number of type 1 infectious individuals. Variable $i_{1}$ (resp. $i_{2}$ ) denotes the number of newly infected individuals by type 1 (resp. type 2) infectious individuals.

$$
\begin{aligned}
& \mathrm{P}\left(X_{t+1}=x(t+1), Y_{t+1}=y(t+1) \mid X_{t}=x(t), Y_{t}=y(t)\right)= \\
& \sum_{i=0}^{y(t)} \sum_{i_{1}=0}^{i k} \sum_{i_{2}=0}^{(y(t)-i) k} 1_{\left\{\left(i_{1}+i_{2}\right) k_{c}=y(t+1)\right\}} b\left(i_{1}, i k, p_{1}\right) b\left(i_{2},(y(t)-i) k, p_{2}\right) b(x(t+1)-y(t), x(t), 1-\gamma) b(i, y(t), p)
\end{aligned}
$$

Fig. 6. Logarithmic value of the transition probability.

event $\{C=i\}, I_{1}$ and $I_{2}$ are independent binomial random variables. The mean and variance of $I_{1}+I_{2}$ are

$$
\mathrm{E}\left[I_{1}+I_{2} \mid C=i\right]=i k p_{1}+(y(t)-i) k p_{2}
$$

and

$$
\sigma_{I_{1}+I_{2} \mid C=i}^{2}=i k p_{1}\left(1-p_{1}\right)+(y(t)-i) k p_{2}\left(1-p_{2}\right) .
$$

Define random variable

$$
R=\sum_{j=1}^{I_{1}+I_{2}} K_{j} .
$$

Recall that $k_{c}$ and $\sigma_{c}^{2}$ are the mean and variance of a clique size, defined in (11) and (12). The conditional mean and the conditional variance of the compound random variable $R$ are

$$
\begin{aligned}
\mathrm{E}[R \mid C=i] & =\mathrm{E}\left[I_{1}+I_{2}\right] \mathrm{E}\left[K_{1}\right] \\
& =\left(i k p_{1}+(y(t)-i) k p_{2}\right) k_{c}
\end{aligned}
$$

and

$$
\begin{aligned}
\operatorname{Var}(R \mid C=i)= & \sigma_{c}^{2} \mathrm{E}\left[I_{1}+I_{2} \mid C=i\right]+\left(k_{c}\right)^{2} \sigma_{I_{1}+I_{2} \mid C=i}^{2} \\
= & \left(i k p_{1}+(y(t)-i) k p_{2}\right) \sigma_{c}^{2}+\left(k_{c}\right)^{2} \\
& \quad\left(i k p_{1}\left(1-p_{1}\right)+(y(t)-i) k p_{2}\left(1-p_{2}\right)\right)
\end{aligned}
$$

according to Wald's equations [22, p. 438-439]. Since $y(t)$ is typically very large, $I_{1}$ and $I_{2}$ are also large. We propose to approximate $R$ by a normal random variable. That is,

$$
\begin{aligned}
& \mathrm{P}(R \in(y(t+1)-1 / 2, y(t+1)+1 / 2) \mid C=i) \\
& =\frac{\exp \left(\frac{-(y(t+1)-\mathrm{E}[R \mid C=i])^{2}}{2 \operatorname{Var}(R \mid C=i)}\right)}{\sqrt{2 \pi \operatorname{Var}(R \mid C=i)}} .
\end{aligned}
$$

We further simplify (36) by applying mean-field approximation [11], [23]. We replace $C$ by its mean value, which is $y(t) p$. Replacing $i$ by $y(t) p$ in (34) and 35 and then assuming that $p_{1}$ and $p_{2}$ are small, we reach

$$
\begin{aligned}
\mu & =\mathrm{E}[R] \\
& =y(t)\left(p p_{1}+(1-p) p_{2}\right) k k_{c} \\
& =y(t) R_{0}
\end{aligned}
$$

and

$$
\begin{aligned}
\sigma^{2}= & \operatorname{Var}(R) \\
= & \sigma_{c}^{2} y(t) k\left(p p_{1}+(1-p) p_{2}\right)+\left(k_{c}\right)^{2} y(t) k \\
& \cdot\left(p p_{1}\left(1-p_{1}\right)+(1-p) p_{2}\left(1-p_{2}\right)\right) \\
\approx & \left(\frac{\sigma_{c}^{2}}{k_{c}}+k_{c}\right) y(t) R_{0},
\end{aligned}
$$

where

$$
R_{0}=\left(p p_{1}+(1-p) p_{2}\right) k k_{c}
$$

is the basic reproduction number of this epidemic network. Let

$$
\xi=\frac{\sigma_{c}^{2}}{k_{c}}+k_{c}
$$

and rewrite

$$
\sigma^{2}=\xi y(t) R_{0}
$$

Taking logarithm on (31) and noting 36, we obtain (40). Eq. (40) is displayed in Fig. 7. Since the second term on the right side of 40 is independent of $p$, maximizing the logarithmic 


$$
\begin{aligned}
& \log \mathrm{P}\left(X_{t+1}=x(t+1), Y_{t+1}=y(t+1) \mid X_{t}=x(t), Y_{t}=y(t)\right) \\
& \approx \log \left(\frac{\exp \left(\frac{-(y(t+1)-\mu)^{2}}{2 \sigma^{2}}\right)}{\sqrt{2 \pi \sigma^{2}}}\right)+\log (b(x(t+1)-y(t), x(t), 1-\gamma)) \\
& =\log \left(\frac{\exp \left(\frac{-\left(y(t+1)-y(t) R_{0}\right)^{2}}{2 \xi y(t) R_{0}}\right)}{\sqrt{2 \pi \xi y(t) R_{0}}}\right)+\log (b(x(t+1)-y(t), x(t), 1-\gamma))
\end{aligned}
$$

Fig. 7. Approximation of transition probabilities by normal distributions.

transition probability is equivalent to maximizing the first term on the right side. Define

$$
\begin{aligned}
f\left(R_{0}\right) \stackrel{\text { def }}{=} & -\frac{\left(y(t+1)-y(t) R_{0}\right)^{2}}{2 \xi y(t) R_{0}}-\frac{1}{2} \log (2 \pi \xi y(t)) \\
& -\frac{1}{2} \log \left(R_{0}\right) .
\end{aligned}
$$

We approximate the optimization problem in (14) by the following optimization problem

$$
\hat{p}(t)=\underset{0 \leq p \leq 1}{\operatorname{argmax}} f\left(R_{0}\right)
$$

with constraint $R_{0} \geq 0$. To study the extrema of $f$ we differentiate $f$ once and twice to get

$$
\begin{aligned}
f^{\prime}\left(R_{0}\right) & =\frac{y(t+1)^{2}-y(t)^{2} R_{0}^{2}-\xi y(t) R_{0}}{2 \xi y(t) R_{0}^{2}} \\
f^{\prime \prime}\left(R_{0}\right) & =\frac{\xi y(t) R_{0}-2 y(t+1)^{2}}{2 \xi y(t) R_{0}^{3}} .
\end{aligned}
$$

Note that

$$
f^{\prime}\left(R_{0}\right)=0
$$

has a unique positive root, which is

$$
R_{0}=\frac{-\xi+\sqrt{\xi^{2}+4 y(t+1)^{2}}}{2 y(t)} .
$$

At this root, the second derivative of $f$ is negative. To see this, note that the positive root in 46 satisfies

$$
\xi y(t) R_{0}=y(t+1)^{2}-y(t)^{2} R_{0}^{2} .
$$

Substituting the preceding into (44), we obtain

$$
\begin{aligned}
f^{\prime \prime}\left(R_{0}\right) & =\frac{\xi y(t) R_{0}-2 y(t+1)^{2}}{2 \xi y(t) R_{0}^{3}} \\
& =\frac{-y(t)^{2} R_{0}^{2}-y(t+1)^{2}}{2 \xi y(t) R_{0}^{3}} \\
& <0 .
\end{aligned}
$$

Thus,

$\log \mathrm{P}\left(X_{t+1}=x(t+1), Y_{t+1}=y(t+1) \mid X_{t}=x(t), Y_{t}=y(t)\right)$

achieves maximum when (46) holds. In view of (38), $R_{0}$ is a quadratic function of $p$. We solve $p$ from (46). Recall that we assume (4), (5) and (6). Under these assumptions, (46) can have a unique root of $p$ in $[0,1]$, in which case, the unique root is the solution of the optimization problem (42). Eq. 46) can have no root of $p$ in $[0,1]$, in which case the solution of 42 is $\hat{p}(t)=0$ or $\hat{p}(t)=1$. We summarize the solution of the optimization problem $(42)$ in the following proposition. The proof of the proposition is presented in the appendix at the end of this paper.

Proposition 1. Eq. (46) has two real roots in p. Either (46) has exactly one root in interval $[0,1]$, or it has no root in this interval. If (46) has exactly one root in [0,1], it is the smaller root (denoted by $p^{\star}$ ) of the two roots. In this case, the optimal solution of (42) is $p^{\star}$. If Eq. (46) has no roots in $[0,1]$, either $p^{\star}<0$ or $p^{\star}>1$. In the former case, the optimal solution is $\hat{p}(t)=0$. In the latter case, the optimal solution is $\hat{p}(t)=1$.

\section{A. Clustering Coefficient}

In this section we analyze the clustering coefficient of the social network model described in section III According to Newman [24, Eq. (7.41)], the clustering coefficient of a network is defined as

$$
M=\frac{3 \mathrm{E}[T]}{\mathrm{E}[D]},
$$

where $\mathrm{E}[T]$ is the expected number of triangles, and $\mathrm{E}[D]$ is the expected number of connected triples in the network. To derive these two quantities, let $\left\{K_{i j}\right\}$ be an i.i.d. double sequence of random variables that has the same distribution of $K$. Random variable $\left\{K_{i j}\right\}$ denotes the size of the $j$-th clique in layer $i$. Let $N_{i}$ (resp. $C_{i}$ ) be the number of vertices (resp. cliques) in layer $i$. It is easy to see that $N_{i}$ and $C_{i}$ satisfy the following recursion

$$
\begin{aligned}
N_{i} & =\sum_{j=1}^{C_{i}} K_{i j} \\
C_{i+1} & =k N_{i},
\end{aligned}
$$

starting from $C_{0}=1$ and $N_{0}=K_{01} \cdot N_{i}$ defined in (48) is a compound random variable, whose mean can be evaluated using Wald's equations [22]. That is,

$$
\begin{aligned}
\mathrm{E}\left[N_{i}\right] & =\mathrm{E}\left[C_{i}\right] \mathrm{E}[K]=k_{c} \mathrm{E}\left[C_{i}\right] \\
\mathrm{E}\left[C_{i+1}\right] & =k \mathrm{E}\left[N_{i}\right] .
\end{aligned}
$$


It can be shown by easy induction that the preceding two equations lead to the following

$$
\begin{aligned}
\mathrm{E}\left[N_{i}\right] & =k_{c}\left(k k_{c}\right)^{i} \\
\mathrm{E}\left[C_{i}\right] & =\left(k k_{c}\right)^{i} .
\end{aligned}
$$

We now derive the expected number of triangles and the expected number of connected triples in the social network described in section III Suppose that the network has $L$ layers from indexed from zero to $L-1$. The number of triangles in layer $i$ is

$$
T_{i}=\sum_{j=1}^{N_{i}}\left(\begin{array}{c}
K_{i j} \\
3
\end{array}\right) 1_{\left\{K_{i j} \geq 3\right\}},
$$

$i=0,1, \ldots, L-1$. Since

$$
\mathrm{E}\left[K^{j} \mid K \geq 3\right]=\frac{\sum_{x=3}^{\infty} x^{j} \operatorname{Pr}(K=x)}{\operatorname{Pr}(K \geq 3)}
$$

for any $j \geq 1$, one can express $\mathrm{E}\left[T_{i}\right]$ more compactly in terms of the conditional expectation $\mathrm{E}\left[K^{j} \mid K \geq 3\right]$. Specifically,

$$
\begin{aligned}
\mathrm{E}\left[T_{i}\right]= & \mathrm{E}\left[N_{i}\right] \operatorname{Pr}(K \geq 3) . \\
& \frac{\mathrm{E}\left[K^{3} \mid K \geq 3\right]-3 \mathrm{E}\left[K^{2} \mid K \geq 3\right]+2 \mathrm{E}[K \mid K \geq 3]}{6} .
\end{aligned}
$$

There are four types of connected triples. An example to illustrate the triangles and connected triples is shown in Figure 9 In the first type of connected triples, both edges connect vertices in a clique. The expected number of type 1 connected triples in layer $i$ is

$$
D_{1, i}=\sum_{j=1}^{N_{i}} K_{i j}\left(\begin{array}{c}
K_{i j}-1 \\
2
\end{array}\right) 1_{\left\{K_{i j} \geq 3\right\}}, \quad i=0,1, \ldots, L-1 .
$$

It follows from the same argument leading to 52 that

$$
\begin{aligned}
\mathrm{E}\left[D_{1, i}\right]= & \mathrm{E}\left[N_{i}\right] \operatorname{Pr}(K \geq 3) . \\
& \frac{\mathrm{E}\left[K^{3} \mid K \geq 3\right]-3 \mathrm{E}\left[K^{2} \mid K \geq 3\right]+2 \mathrm{E}[K \mid K \geq 3]}{2} .
\end{aligned}
$$

We have

$$
3 \mathrm{E}\left[T_{i}\right]=\mathrm{E}\left[D_{1, i}\right] .
$$

In the second type of connected triples, one edge connects two vertices in a layer $i$ clique, and the second edge connects the two vertices with a vertex in layer $i+1$. The number of type 2 connected triples is

$$
D_{2, i}=\sum_{j=1}^{N_{i}} K_{i j}\left(K_{i j}-1\right) k, i=0,1, \ldots, L-2 .
$$

It follows that

$$
\mathrm{E}\left[D_{2, i}\right]=\mathrm{E}\left[N_{i}\right]\left(\mathrm{E}\left[K^{2}\right]-\mathrm{E}[K]\right) k .
$$

A third type of connected triple connects a vertex in layer $i$ with two vertices in layer $i-1$ and $i+1$. The number of type 3 connected triples is

$$
D_{3, i}=k N_{i}, \quad i=1,2, \ldots, L-2 .
$$

It follows that

$$
\mathrm{E}\left[D_{3, i}\right]=k \mathrm{E}\left[N_{i}\right] .
$$

In the fourth type of connected triples, one edge connects two vertices in a layer $i$ clique, and the second edge connects the two vertices with a vertex in layer $i-1$. The number of type 4 connected triples is

$$
D_{4, i}=\sum_{j=1}^{N_{i}}\left(K_{i j}-1\right), \quad i=1,2, \ldots, L-1 .
$$

It follows that

$$
\mathrm{E}\left[D_{4, i}\right]=\mathrm{E}\left[N_{i}\right](\mathrm{E}[K]-1) .
$$

Substituting (52), (53), (54), (55) and (56) into (47), we have (57) displayed in Fig. 8

In a special case, in which $K$ is degenerate and is equal to $k_{c}$, one can further simplify (57). Particularly, letting $L \rightarrow \infty$, one obtains

$$
M \rightarrow \begin{cases}\frac{k_{c}^{2}\left(k_{c}-1\right)\left(k_{c}-2\right)}{k_{c}^{2}\left(k_{c}-1\right)\left(k_{c}-2\right)+4 k_{c}\left(k_{c}-1\right)+2} & k_{c} \geq 3 \\ 0 & k_{c}=1,2 .\end{cases}
$$

\section{Percolation Analysis}

In this section we consider a random contact network, in which a disease transmitted by droplets or aerosols spreads according to an independent cascade model. That is, the disease transmits from a node at one end of an edge to the node at the other end with a probability. In addition, the transmissions along all edges are independent. We shall present a percolation analysis of this model and obtain percolation thresholds and sizes of giant components.

We now describe our model. Consider a random graph $(G, V, E)$. Randomly select a node from the graph. Let $Z$ be the degree of this node. Let $g_{0}(z)$ denote the probability generating function of $Z$, i.e.,

$$
g_{0}(z)=\sum_{k=0}^{\infty} \mathrm{P}(Z=k) z^{k} .
$$

Now randomly select an edge. Let $Y$ be the excess degree of a node reached along the randomly selected edge. Let $g_{1}(z)$ be the probability generating function of $Y$, i.e.

$$
g_{1}(z)=\sum_{k=0}^{\infty} \mathrm{P}(Y=k) z^{k}
$$

Every node in this graph can be one of two types. A type 1 node denotes an individual who wears a facial mask and a type 2 node denotes an individual who does not. A randomly selected node is of type 1 with probability $p$ and is of type 2 with probability $1-p$. Assume that this event is independent of anything else. As mentioned before, an infectious disease spreads in this network according to an independent cascade model. Consider a randomly selected edge connecting two nodes, say node $V_{1}$ and node $V_{2}$. Let $\phi_{i j}$ be the conditional probability that the disease transmits from node $V_{1}$ to node 


$$
M=\frac{3 \sum_{\ell=0}^{L-1} \mathrm{E}\left[T_{i}\right]}{3 \sum_{\ell=0}^{L-1} \mathrm{E}\left[T_{i}\right]+\sum_{i=0}^{L-2} \mathrm{E}\left[D_{2, i}\right]+\sum_{i=1}^{L-2} \mathrm{E}\left[D_{3, i}\right]+\sum_{i=1}^{L-1} \mathrm{E}\left[D_{4, i}\right]} .
$$

Fig. 8. Clustering coefficient.

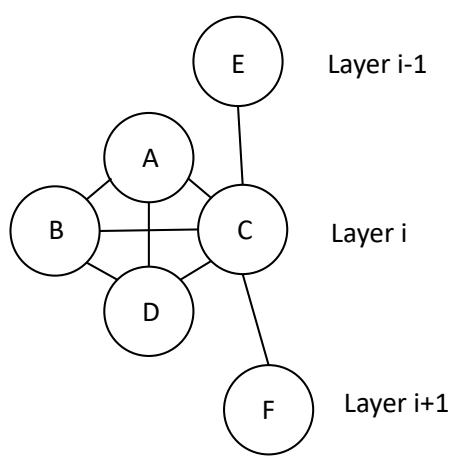

Fig. 9. An illustration of triangles and connected triples. In this example, $k_{c}=4$ and $k$ is not shown. Vertices $A, B, C$ and $D$ form a clique in layer $i$. Vertices $A, B$ and $C$ form a triangle. $B A C$ is a type one connected triple. $D C F$ is a type two connected triple. $E C F$ is a type three connected triple and $E C D$ is a type four connected triple.

$V_{2}$, given that the types of nodes $V_{1}$ and $V_{2}$ are $i$ and $j$, respectively, where $i, j=1,2$.

We now present a percolation analysis of the random network model described above. Percolation analysis has been a useful tool to study resilience of communication networks [25], [26] and epidemic networks [27]-[30]. There are two types of percolation models, bond percolation and site percolation, depending on whether edges or nodes, respectively, are removed randomly [11]. Our model is a form of bond percolation, where edges are randomly removed. Removed edges imply that diseases can not be transmitted from one end of these edges to the other end. The size of the largest component in the percolated network is the maximum fraction of possibly infected population in the network.

Randomly select an edge. Let $V_{1}$ and $V_{2}$ be the two nodes at the two ends of the edge. Suppose that the type of node $V_{1}$ is $i$. Let $E_{i}$ be the event that along the selected edge from $V_{1}$, one can not reach a giant component. Let $u_{i}=\mathrm{P}\left(E_{i}\right)$. Now we condition on the event that the type of node $V_{1}$ is $j$. Event $E_{i}$ will occur, if the randomly selected edge is removed. This occurs with probability $1-\phi_{i j}$. With probability $\phi_{i j}$ the randomly selected edge is present. Let $Y$ be the number of neighbors of node $V_{2}$, not including $V_{1}$. Event $E_{i}$ will occur, if one can not reach a giant component along any one these $Y$ edges. Combining these arguments, we have

$$
\begin{aligned}
u_{i}= & p\left(1-\phi_{i 1}+\phi_{i 1} \sum_{k=0}^{\infty} \mathrm{P}\left(E_{1} \mid Y=k\right) \mathrm{P}(Y=k)\right) \\
& +(1-p)\left(1-\phi_{i 2}+\phi_{i 2} \sum_{k=0}^{\infty} \mathrm{P}\left(E_{2} \mid Y=k\right) \mathrm{P}(Y=k)\right)
\end{aligned}
$$

$$
\begin{aligned}
& =p\left(1-\phi_{i 1}+\phi_{i 1} \sum_{k=0}^{\infty} \sum_{\ell=0}^{k} \mathrm{P}\left(E_{1} \mid Y=k, C=\ell\right)\right. \\
& \quad \times \mathrm{P}(C=\ell \mid Y=k) \mathrm{P}(Y=k)) \\
& +(1-p)\left(1-\phi_{i 2}+\phi_{i 2} \sum_{k=0}^{\infty} \sum_{\ell=0}^{k} \mathrm{P}\left(E_{2} \mid Y=k, C=\ell\right)\right. \\
& \quad \times \mathrm{P}(C=\ell \mid Y=k) \mathrm{P}(Y=k)),
\end{aligned}
$$

where $C$ is the number of type 1 nodes connected to node $V_{2}$ not including $V_{1}$. The distribution of $C$ conditioning on $Y=k$ is binomial. Thus, Eq. (59) becomes

$$
\begin{aligned}
u_{i}= & p\left(1-\phi_{i 1}+\phi_{i 1} \sum_{k=0}^{\infty} \sum_{\ell=0}^{k}\left(u_{1}^{\ell} \cdot u_{2}^{k-\ell}\right)\right. \\
& \left.\times\left(\begin{array}{c}
k \\
\ell
\end{array}\right) p^{\ell}(1-p)^{k-\ell} \mathrm{P}(Y=k)\right) \\
& +(1-p)\left(1-\phi_{i 2}+\phi_{i 2} \sum_{k=0}^{\infty} \sum_{\ell=0}^{k}\left(u_{1}^{\ell} \cdot u_{2}^{k-\ell}\right)\right. \\
& \left.\quad \times\left(\begin{array}{c}
k \\
\ell
\end{array}\right) p^{\ell}(1-p)^{k-\ell} \mathrm{P}(Y=k)\right) \\
= & p\left(1-\phi_{i 1}+\phi_{i 1} g_{1}\left(p u_{1}+(1-p) u_{2}\right)\right) \\
& +(1-p)\left(1-\phi_{i 2}+\phi_{i 2} g_{1}\left(p u_{1}+(1-p) u_{2}\right)\right) \\
= & 1-\left(p \phi_{i 1}+(1-p) \phi_{i 2}\right)\left(1-g_{1}\left(p u_{1}+(1-p) u_{2}\right)\right)
\end{aligned}
$$

for $i=1,2$. Eq. (60) is a system of nonlinear equations, from which we can solve for $u_{1}$ and $u_{2}$.

Once we have $u_{1}$ and $u_{2}$ we can compute the giant component size of the percolated network. Randomly select a node from the network. Let $S_{i}$, where $i=1,2$, be the conditional probability that the randomly selected node is connected with a giant component, given that the selected node is of type $i$. Let $X$ be the degree of the randomly selected node. The node is connected with a giant component if along at least one of its edges one can reach a giant component. Conditioning on $X=k$, let $I$ be the number of type 1 nodes among the $k$ neighbors. Combining all these arguments, we have

$$
\begin{aligned}
S_{i} & =1-\sum_{k=0}^{\infty} \sum_{j=0}^{k} u_{1}^{j} u_{2}^{k-j} \mathrm{P}(X=k)\left(\begin{array}{c}
k \\
j
\end{array}\right) p^{j}(1-p)^{k-j} \\
& =1-g_{0}\left(p u_{1}+(1-p) u_{2}\right)
\end{aligned}
$$


for $i=1,2$. By taking average on the conditional probabilities, a randomly selected node is connected to a giant component with probability

$$
S=p S_{1}+(1-p) S_{2} .
$$

This is also the expected size of the giant component.

\section{A. Percolation Threshold}

Let $\boldsymbol{u}$ be a $2 \times 1$ vector over the set of real numbers $\mathcal{R}$. Specifically, let

$$
\boldsymbol{u}=\left(u_{1}, u_{2}\right)^{T},
$$

where symbol $T$ denotes transposition of vectors. Let $\boldsymbol{f}$ be a vector-valued function mapping from $\mathcal{R}^{2}$ to $\mathcal{R}^{2}$, i.e.,

$$
\begin{aligned}
& \boldsymbol{f}(\boldsymbol{x})=\left(f_{1}(\boldsymbol{x}), f_{2}(\boldsymbol{x})\right)^{T}= \\
& \left(\begin{array}{c}
1-\left(p \phi_{11}+(1-p) \phi_{11}\right)\left(1-g_{1}\left(p x_{1}+(1-p) x_{2}\right)\right) \\
1-\left(p \phi_{21}+(1-p) \phi_{22}\right)\left(1-g_{1}\left(p x_{1}+(1-p) x_{2}\right)\right)
\end{array}\right),
\end{aligned}
$$

where $\boldsymbol{x}=\left(x_{1}, x_{2}\right)$. Eq. 60 implies that $\boldsymbol{u}$ is a root of

$$
\boldsymbol{x}=\boldsymbol{f}(\boldsymbol{x})
$$

The roots of equations of the form 63 are also called the fixed points of function $f$. It is clear that function $f$ always has fixed point $\mathbf{1}=(1,1)^{T}$. Lee et al. [31] established that $\boldsymbol{f}$ has an additional fixed point if the dominant eigenvalue of the Jacobian matrix evaluated at $\mathbf{1}$ is greater than one. In addition, this fixed point is attractive. The Jacobian matrix for function $\boldsymbol{f}$ evaluated at $\boldsymbol{x}=\boldsymbol{a}$ is defined as

$$
\left.\left(\begin{array}{ll}
\frac{\partial f_{1}(\boldsymbol{x})}{\partial x_{1}} & \frac{\partial f_{1}(\boldsymbol{x})}{\partial x_{2}} \\
\frac{\partial f_{2}(\boldsymbol{x})}{\partial x_{1}} & \frac{\partial f_{2}(\boldsymbol{x})}{\partial x_{2}}
\end{array}\right)\right|_{\boldsymbol{x}=\mathbf{a}}
$$

The Jacobian matrix for the function $f$ defined in 62 evaluated at $(1,1)^{T}$ is

$$
\boldsymbol{J}=\mathrm{E}[Y]\left(\begin{array}{ll}
\phi_{11} p & \phi_{12}(1-p) \\
\phi_{21} p & \phi_{22}(1-p)
\end{array}\right)
$$

where $\mathrm{E}[Y]=g_{1}^{\prime}(1)$ is the expected excess degree of a node reached by a randomly selected edge. It is easy to derive the eigenvalues of $\boldsymbol{J}$. Denote the two eigenvalues of $\boldsymbol{J}$ by $\lambda_{1}$ and $\lambda_{2}$, Then,

$$
\begin{aligned}
& \lambda_{1}=\frac{\mathrm{E}[Y]}{2}\left(p \phi_{11}+(1-p) \phi_{22}+\sqrt{\Delta}\right) \\
& \lambda_{2}=\frac{\mathrm{E}[Y]}{2}\left(p \phi_{11}+(1-p) \phi_{22}-\sqrt{\Delta}\right)
\end{aligned}
$$

where

$$
\Delta=\left(p \phi_{11}-(1-p) \phi_{22}\right)^{2}+4(1-p) p \phi_{21} \phi_{12} .
$$

Note that both eigenvalues are real, and $\lambda_{1}>\lambda_{2}$. Thus, spectral radius or the dominant eigenvalue of $\boldsymbol{J}$ is $\lambda_{1}$. It is also this eigenvalue that controls the percolation threshold of the epidemic network.

\section{Numerical and Simulation Results}

In this section we present numerical and simulation results. We first verify the accuracy of (40) to approximate the transition probability in 29]. We select an arbitrary set of parameters:

$$
k=3, x_{0}=5, \phi_{22}=0.8 .
$$

In this section, we assume that $K$ is deterministic and $K=k_{c}$. According to [2], the efficiency of a typical mask is in the range from $0.58 \%$ to $85 \%$. Since masks are typically more efficient to stop viral transmission if sources wear masks [4], we set $\eta_{1}=0.8$ and $\eta_{2}=0.65$. We select a value for $p$ between 0 and 1 . We randomly generate ten thousand values for $y(t)$. We perform simulation to generate ten thousand values for $y(t+1)$ based on $y(t)$ and $p$. We solve (14) based on (32) and (42) for ten thousand values of $y(t)$ and $y(t+1)$. We calculate the average distance between the two solutions for $k_{c}=1$ and $k_{c}=3$. The result is shown in Figure 10 This shows that the approximation method works very well for $k_{c}=1$. The approximation error for $k_{c}=3$ is also acceptable.

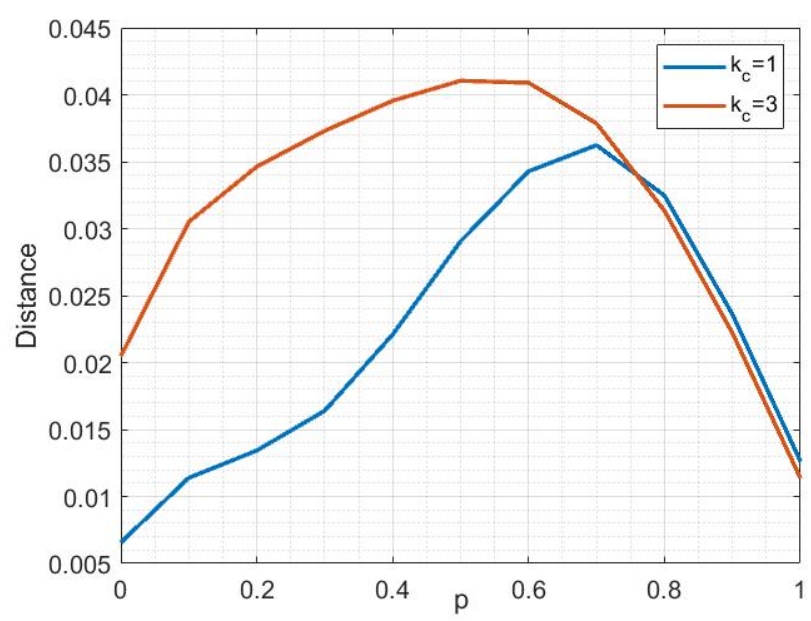

Fig. 10. Distance between the solution of the constraint optimization using exact transition probability in 32 and the approximated transition probability in 42.

Next, we use the data published by John Hopkins University [5] to predict the spread of the COVID-19 pandemic using our model. In this study, we assume that $K$ is deterministic and $K=k_{c}=3$. Due to large infection rates of some countries, we assume that $k=\mathrm{E}[Y]=150$ in order to have $\phi_{22} \leq 1$. We choose $\tau=5$ days as the width of a time slot in the time dependent SIR model. We first execute Algorithm 1 on the number of infected and recovered individuals in mainland China. The time functions of $\beta_{22}(t)$ and $p(t)$ are shown in Figure 11. The maximum fraction of population who will be infected predicted at time $t$ is also shown in the figure. The epidemic started from the People's Republic of China from January, 2020. The authority managed to contain the epidemic very well by the end of February. However, there are several 
significant events between January and August of 2020. In mid April, the Chinese authority revised the way that death toll is calculated [32]. In mid June, Beijing faced a second wave of infections [33] In mid July, there was a surge of infected number of individuals in Xinjiang [34]. At the end of July, there was a surge in Dalian [35]. From Figure 11, we see that $\beta_{22}(t)$ is large initially in January, and is controlled to reach a small value by the end of February. The value of $\beta_{22}(t)$ again rises and drops before and after the events described above. From Figure 11 , note that $p(t)$ also rises and decreases before and after the events. However, function $p(t)$ lags behind function $\beta_{22}(t)$. This correlation has a nice interpretation. Rise in $\beta_{22}(t)$ usually manifests itself in the rise of infected number of individuals. As the population sees a rise in the infected number of individuals, more people wear masks to protect themselves. On the other hand, decreases of $\beta_{22}(t)$ result in less number of newly infected individuals. People typically see this as sign of a safe community. Thus, less people wear masks in public places. As $\beta_{22}(t)$ rises, the predicted size of giant component $S(t)$ also increases. However, as $p(t)$ catches up and rises, $S(t)$ decreases. Notice that the time sequence $\beta_{22}(t)$ reflects a joint efforts of many measures to contain the epidemic. Wearing facial masks is a measure in the personal level. Shutting down schools, businesses, and keeping people at home is a measure in the government level. In this paper, we consider only the measure of wearing masks and ignore other measures. Thus, $p(t)$ in Figure 11 may be higher than the actual fraction of population who wear masks, as it reflects a joint effect of many measures to contain the epidemic. Note that the value of $k_{c}$ can influence the value of $p(t), \beta_{22}(t)$ and the predicted size of giant components $S(t)$. In Figure 12 we show the time function $p(t)$ for $k_{c}=3$ and $k_{c}=1$. The more densely clustered people are, the larger $k_{c}$ is and the more infectious the disease is. From Figure 12, we see that population react and more people wear masks if $k_{c}$ is large.

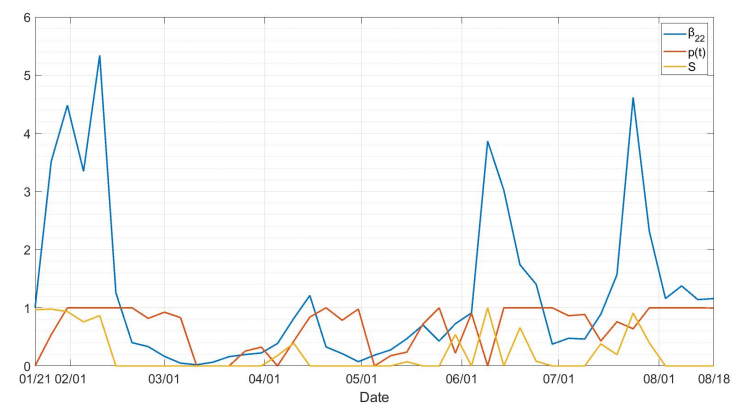

Fig. 11. Time functions $\beta_{22}(t), p(t), S(t)$ of mainland China.

Next we study the epidemics of six countries. They are the United States of America, India, France, Italy, South Korea and Japan. We execute Algorithm 1 to compute $\beta_{22}(t), p(t)$ and $S(t)$. The time functions of $\beta_{22}(t), p(t)$ and $S(t)$ for the six countries are shown in Figure 13 . Figure 14 and Figure 15.

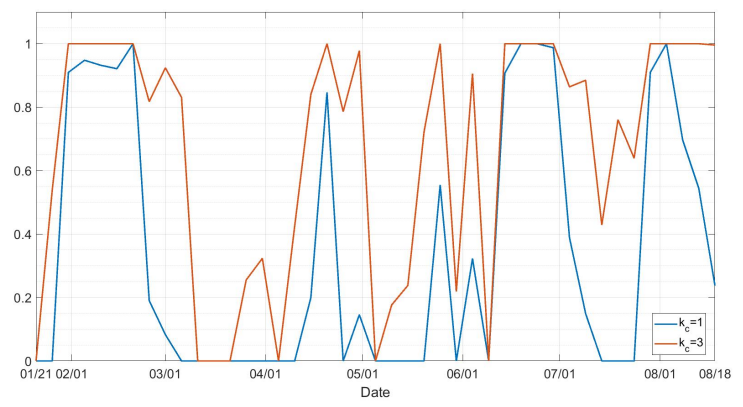

Fig. 12. $p(t)$ of mainland China from Jan. 21st to Aug. 18, 2020 for $k_{c}=1$ and $k_{c}=3$.

respectively. We present $p(t)$ and $S(t)$ of the six countries in Figure 14 and Figure 15. Since these functions fluctuate a lot, we use a built-in Matlab function to compute the polynomial regression of these functions in order to show a general trend. Note that India has a smaller infection rate $\beta_{22}(t)$ than that of the U.S.A. However, India has a much smaller predicted size of giant component than that of the U.S.A. This is because India has a very large recovery rate [36], [37]. We also note that around July France has a $\beta_{22}(t)$ comparable with that of the US. However, France has a high predicted $S(t)$ after August. It is worth noting that $p(t)$ of France in mid August is not close to 1 . By raising $p(t)$, France may have a better control of the epidemics. Finally, we show the average value of $p(t)$ for three western countries versus that of three Asian countries. The average value of $p(t)$ of western countries and Asian countries rises nearly equally quickly in the beginning of March 2020. Asian countries had a better control of the epidemic. As a result, Asian people relaxed and $p(t)$ was significantly lower in April and May 2020. The rise and drop of $p(t)$ for Japan and South Korea in Figure 14 and Figure 16 during April and May 2020 seem to agree with the timing of some major outbreaks recorded in [38], [39].

Finally, we compare the size of giant components obtained in 61 with that obtained by simulation of a few well-known data sets [40]. The result is shown in Table $\mathrm{V}$. In this study, we set $p=0.4$ and $\phi_{22}=0.6$. In each simulation, we randomly select a vertex and then randomly select two vertices that are distance $i$ from the vertex for $i=1,2, \ldots, 5$. These eleven individuals are infected at time zero. We simulate the independent cascade model until no new infection is observed. We repeat the simulation fifty times before we take an average. From Table V, we note that simulation results of real-life networks are consistently smaller than those obtained from 61. Note also that real-life social networks nearly always possess clustering and community structures, while the random network model that leads to 61 does not. Dense connections within a community are helpful to the spreading of the disease within the community [18], [19]. However, community boundaries can hinder the spreading of the disease. 


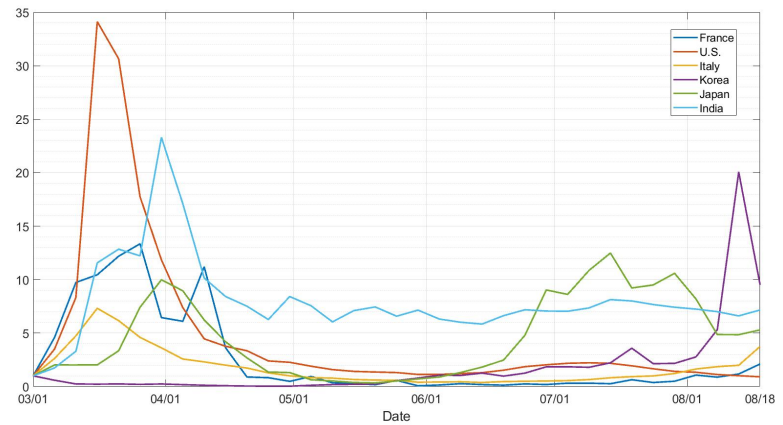

Fig. 13. $\beta_{22}(t)$ of the U.S., India, France, Italy, Korea and Japan.

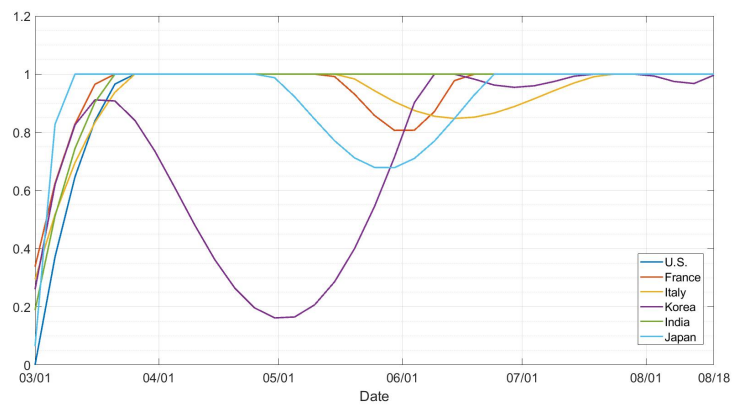

Fig. 14. $p(t)$ of the U.S., India, France, Italy, Korea and Japan.

\section{Vi. CONCLusions}

In this paper, we presented a time dependent SIR model, in which some individuals wear facial masks and some do not. Based on the number of infected individuals and the number of recovered individuals published by J. H. University, we estimate the disease infection rates and recovery rates. We proposed a probabilistic version of the SIR model. We derived the transition probability of this random SIR model. By maximizing the transition probability, we estimate the most probable value of the fraction of population who wear masks. This transition probability numerically difficult to compute,

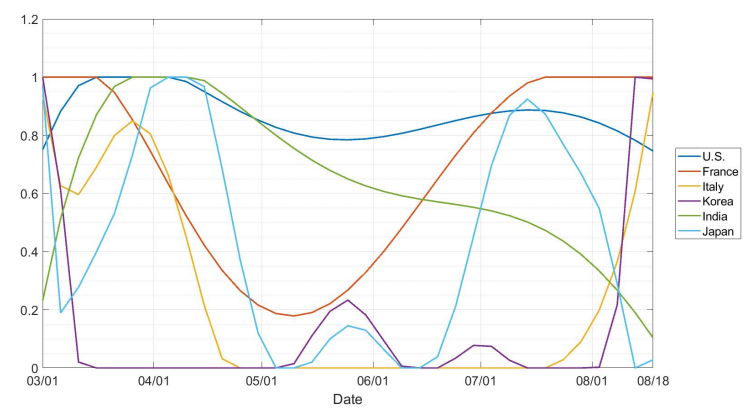

Fig. 15. Predicted size of giant component of the U.S., India, France, Italy, Korea and Japan.

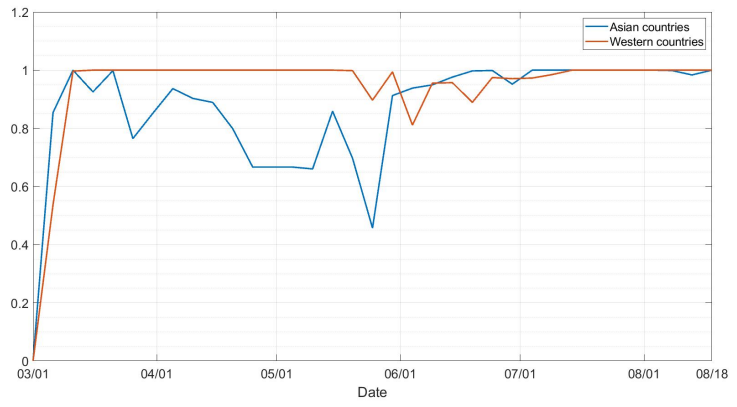

Fig. 16. Average value of $p(t)$ of three western counties versus that of three Asian countries.

\begin{tabular}{|l|r|r|r|r|}
\hline network & $\begin{array}{r}\text { number of } \\
\text { vertices }\end{array}$ & $\begin{array}{r}\text { number of } \\
\text { edges }\end{array}$ & $r(\infty)$ & $S$ \\
\hline \hline email-Eu-core & 1005 & 25571 & 0.782 & 0.862 \\
\hline Facebook & 4039 & 88234 & 0.744 & 0.898 \\
\hline twitch-ES & 4648 & 59382 & 0.724 & 0.803 \\
\hline twitch-EN & 7126 & 35324 & 0.571 & 0.640 \\
\hline lastfm asia & 7624 & 27806 & 0.490 & 0.577 \\
\hline ca-CondMat & 23133 & 93497 & 0.535 & 0.645 \\
\hline deezer_europe & 28281 & 92752 & 0.500 & 0.579 \\
\hline
\end{tabular}

TABLE I

SIR SPREADING IN EIGHT REAL-LIFE NETWORKS. SYMBOL $r(\infty)$ DENOTES THE FRACTION OF VERTICES THAT ARE EVER INFECTED BY THE DISEASE IN SIMULATION. $S$ IS THE CORRESPONDING VALUE OBTAINED BY CALCULATING EQ. 61,.

if the states of model are large. Based on central limit theorem, we proposed an approximation. Through numerical and simulation study, we show that the approximation works well. Finally we carried out a percolation analysis to predict the eventual fraction of population who will be infected with the disease. We proposed a progressive analysis of the epidemics. Using results from the progressive analysis, we analyzed the epidemics of six countries.

\section{Appendix}

In this appendix, we prove Proposition 1

Proof of Proposition 1. We rewrite $R_{0}$ defined in (38) to show dependency with $p$ explicitly, i.e.

$$
R_{0}(p)=k k_{c} \phi_{22}\left(\eta_{1} \eta_{2} p^{2}-\left(\eta_{1}+\eta_{2}\right) p+1\right) .
$$

In (67) we have assumed (4), (5) and (6). Note that $\eta_{1}<1$ and $\eta_{2}<1$. Since the right side of 67 is a quadratic polynomial in $p$, it is easy to establish the following results.

1) $R_{0}(0)>R_{0}(1)>0$.

2) $R_{0}^{\prime}(0)=k k_{c} \phi_{22}\left(-\eta_{1}-\eta_{2}\right)<0$.

3) $R_{0}^{\prime}(1)=k k_{c} \phi_{22}\left(\eta_{1}\left(\eta_{2}-1\right)+\eta_{2}\left(\eta_{1}-1\right)\right)<0$.

4) The minimum of $R_{0}(p)$ occurs at

$$
p=\frac{1}{2 \eta_{1}}+\frac{1}{2 \eta_{2}} .
$$

This point is greater than one, since $\eta_{1}<1$ and $\eta_{2}<1$.

5) The minimum of $R_{0}(p)$ is

$$
-k k_{c} \phi_{22} \frac{\eta_{1} \eta_{2}\left(\eta_{1}-\eta_{2}\right)^{2}}{4 \eta_{1}^{2} \eta_{2}^{2}}
$$


which is negative.

From the results above and the fact that the right side of 46 is positive, it follows that 46 must have two real roots. It also follows from the results above that $R_{0}(p)$ is monotonically decreasing and 46 can not have two roots in $[0,1]$. Since $R_{0}(p)$ is decreasing for $p \in[0,1]$, it follows that if 46 has exactly one root in $[0,1]$, the root is $p^{\star}$. It is also quite clear that if (46) has a unique root in $[0,1]$, the root is the optimal solution of (42).

Now we analyze cases, in which (46) does not have roots in $[0,1]$. Since $R_{0}(p)$ is decreasing for $p \in[0,1]$, it follows that either both roots of (46) are greater than 1 , or $p^{\star}<0$ and the other root is greater than 1 . We now analyze these two cases separately. We first note that we have treated $R_{0}$ as an independent variable in (42). Thus, the derivative $f^{\prime}\left(R_{0}\right)$ is with respect to $R_{0}$. In the two cases in which Eq. (46) has no roots in $[0,1]$, we have to treat the objective function in 42 as a function of $p$. Specifically, the derivative of the objective function with respect to $p$ is

$$
\frac{d}{d p} f\left(R_{0}(p)\right)=f^{\prime}\left(R_{0}\right) R_{0}^{\prime}(p)
$$

where $f^{\prime}\left(R_{0}\right)$ is given in 43 . We claim that in the first case the objective function of $(42)$ is increasing for all $p \in[0,1]$. Thus, the optimal solution of 42 is $\hat{p}(t)=1$. We also claim that in the second case, the objective function of (42) is decreasing for all $p \in[0,1]$. Thus, the optimal solution of (42) is $\hat{p}(t)=0$.

We now prove the two claims. From (43) and 68,

$$
\frac{d}{d p} f\left(R_{0}(p)\right)=\frac{h\left(R_{0}\right) R_{0}^{\prime}(p)}{2 \xi y(t) R_{0}^{2}},
$$

where

$$
h\left(R_{0}\right)=y(t+1)^{2}-y(t)^{2} R_{0}^{2}-\xi y(t) R_{0} .
$$

Note that $h$ is a quadratic and concave function of $R_{0}$ with $h(0)=y(t+1)^{2}>0$. It is clear that equation $h\left(R_{0}\right)=0$ has two real roots. Function $h$ has a zero at $R_{0}=c$, where $c$ is the right side of (46), i.e.

$$
c=\frac{-\xi+\sqrt{\xi^{2}+4 y(t+1)^{2}}}{2 y(t)} .
$$

If $R_{0}(1)<c<R_{0}(0)$, equation $h\left(R_{0}\right)=0$ has exactly one root in the closed interval $[0,1]$. This root is $R_{0}=c$. If $c<R_{0}(1)$, both roots of 46 are greater than 1 . This corresponds to the first case. If $c>R_{0}(0)$, then $p^{\star}<0$ and the other root is greater than 1 . This corresponds to the second case. In the first case,

$$
R_{0}(p)>c
$$

for all $p \in[0,1]$. Thus,

$$
h\left(R_{0}(p)\right)<h(c)=0
$$

for all $p \in[0,1]$. From 69, it follows that $d f\left(R_{0}(p)\right) / d p>0$, since $R_{0}^{\prime}(p)<0$ for all $p \in[0,1]$. This proves the first claim. In the second case,

$$
R_{0}(p)<c
$$

for all $p \in[0,1]$. Thus,

$$
h\left(R_{0}(p)\right)>h(c)=0
$$

for all $p \in[0,1]$. From 69 , it follows that $d f\left(R_{0}(p)\right) / d p<0$. This proves the second claim.

\section{REFERENCES}

[1] A. C. K. Lai, C. K. M. Poon, and A. C. T. Cheung, "Effectiveness of facemasks to reduce exposure hazards for airborne infections among general populations," Journal of the Royal Society Interface, vol. 9, no. 70, p. 938, 2012.

[2] N. C. J. Brienen, A. Timen, J. Wallinga, J. E. V. Steenbergen, and P. F. M. Teunis, "The effect of mask use on the spread of influenza during a pandemic," Risk Analysis, vol. 30, no. 8, pp. 1210-1218, 2010.

[3] A. Davies, K.-A. Thompson, K. Giri, G. Kafatos, J. Walker, and A. Bennett, "Testing the efficacy of homemade masks: Would they protect in an influenza pandemic?" Disaster medicine and public health preparedness, vol. 7, pp. 413-418, August 2013.

[4] R. B. Patel, S. D. Skaria, M. M. Mansour, and G. C. Smaldone, "Respiratory source control using a surgical mask: An in vitro study," Journal of Occupational \& Environmental Hygiene, 2016.

[5] CSSEGISandData and J. H. University, Covid-19, 2020. [Online]. Available: https://github.com/CSSEGISandData/COVID-19

[6] D. Kempe, J. Kleinberg, and E. Tardos, "Maximizing the spread of influence through a social network," in The ninth ACM SIGKDD international conference on Knowledge discovery and data mining, 2003, pp. 137-146.

[7] D. Easley and J. Kleinberg, Networks, crowds and markets reasoning about a highly connected world. Cambridge University Press, 2010.

[8] W. Li, J. Zhou, and J. Lu, "The effect of behavior of wearing masks on epidemic dynamics," no. 1-7, June 2020.

[9] O. Damette, "Zorro versus covid-19: fighting the pandemic with face masks," preprint, medRxiv, December 2020.

[10] C. Betscha, L. Korn, P. Sprengholz, L. Felgendreff, P. S. S. Eitze, and R. Böhm, "Social and behavioral consequences of mask policies during the covid-19 pandemic," PNAS, vol. 117, no. 36, September 2020.

[11] M. Newman, Networks: An Introduction. New York: Oxford University Press, 2010

[12] M. Opuszko and J. Ruhland, "Impact of the network structure on the sir model spreading phenomena in online networks," Nice, France, 2013.

[13] D. Bernardes, M. Latapy, and F. Tarissan, "Relevance of sir model for real-world spreading phenomena: Experiments on a large-scale p2p system," Istanbul, Turkey, pp. 327-334, August 2012.

[14] Z. Wang, Q. Guo, S. Sun, and C. Xia, "The impact of awareness diffusion on sir-like epidemics in multiplex networks," Applied Mathematics and Computation, vol. 349, pp. 134-147, May 2019.

[15] C. Zheng, C. Xia, Q. Guo, and M. Dehmer, "Interplay between sirbased disease spreading and awareness diffusion on multiplex networks," Journal of Parallel and Distributed Computing, vol. 115, pp. 20-28, May 2018.

[16] E. Coupechoux and M. Lelarge, "How clustering affects epidemics in random networks," Adv. in Appl. Probab., vol. 46, no. 4, pp. 985-1008, December 2014.

[17] P. Trapman, "On analytical approaches to epidemics on networks," Theoretical Population Biology, vol. 71, no. 2, pp. 160 - 173, 2007.

[18] T. House and M. Keeling, "Deterministic epidemic models with explicit household structure," Math. Biosci., vol. 213, no. 1, pp. 29-39, May 2008.

[19] — , "Household structure and infectious disease transmission," Epidemiol. Infect., vol. 137, no. 5, pp. 654-661, May 2009.

[20] B. Rockx and et. al., "Comparative pathogenesis of covid-19, mers, and sars in a nonhuman primate model," Science, vol. 368, pp. 1012-1015, 52020.

[21] Z. Du and et. al., "The serial interval of covid-19 from publicly reported confirmed cases," preprint, medRxiv, May 2020.

[22] S. Ghahramani, Fundamentals of Probability with Stochastic Processes, 3rd ed. Pearson Prentice Hall, 2005.

[23] A. Wipf, "Statistical approach to quantum field theory : An introduction," Lect.Notes Phys., vol. 864, pp. 1-390, Nov 2012.

[24] M. Newman and D. Watts, "Scaling and percolation in the small-world network model," Phys. Rev. E, vol. 60, pp. 7332-7342, 2000. 
[25] R. Cohen, K. Erez, D. ben Avraham, and S. Havlin, "Resilence of the internet to random breakdowns," Phys. Rev. Lett., vol. 85, pp. 4626$4628,2000$.

[26] Y. Hu, B. Ksherim, R. Cohen, and S. Havlin, "Percolation in interdependent and interconnected networks: Abrupt change from second- to first-order transitions," Phys. Rev. E., vol. 84, p. 066116, 2011.

[27] L. Sander, C. Warren, I. Sokolov, C. Simon, and J. Koopman, "Percolation on heterogeneous networks as a model for epidemics," Mathematical Biosciences, vol. 80, pp. 293-305, November-December 2002.

[28] C. Moore and M. Newman, "Epidemics and percolation in small-world networks," Phys. Rev. E, vol. 61, p. 5678, 2000.

[29] J. Cardy and P. Grassberger, "Epidemic models and percolation," J. Phys. A: Math. Gen., vol. 18, no. 6, 1985.

[30] M. Á. Serrano and M. Boguná, "Percolation and epidemic thresholds in clustered networks," Physical review letters, vol. 97, no. 8, p. 088701, 2006.

[31] D.-S. Lee, C.-S. Chang, M. Zhu, and H.-C. Li, "A generalized configuration model with degree correlations and its percolation analysis," Applied Network Science, vol. 4, no. 1, p. 124, Dec 2019. [Online]. Available: https://doi.org/10.1007/s41109-019-0240-2

[32] The death toll in Wuhan was revised from 2579 to 3869 (4/17), 2020. [Online]. Available: http://www.china.org.cn/china/Off_the_Wire/ 2020-04/17/content 75943843.htm

[33] Wikipedia, COVID-19 pandemic in Beijing, 2020. [Online]. Available: ] https://en.wikipedia.org/wiki/COVID-19_pandemic_in_Beijing\#June

[34] , COVID-19 pandemic in Xinjiang, 2020. [Online]. Available: https: //en.wikipedia.org/wiki/COVID-19_pandemic_in_Xinjiang\#July_2020

[35] en.people.cn, Officials rule out domestic transmission as origin of Dalian COVID-19 cluster, 2020. [Online]. Available: http: //en.people.cn/n3/2020/0803/c90000-9717687.html

[36] T. of India, Coronavirus recovery: What do India's high COVID recovery numbers really mean? We explain, 2020. [Online]. Available: https://timesofindia.indiatimes.com/life-style/health-fitness/health-new s/coronavirus-recovery-what-do-indias-high-covid-recovery-numbers-r eally-mean-we-explain/photostory/78102510.cms

[37] H. Times, India's Covid-19 recovery rate surges past $78 \%$, one of the highest globally: Govt, 2020. [Online]. Available: https://www.hindustantimes.com/india-news/india-s-covid-19-recover y-rate-surges-past-78-one-of-the-highest-globally-govt/story-wU8YaD W6qG6WEBieTzoD7J.html

[38] Wikipedia, COVID-19 pandemic in South Korea, 2020. [Online]. Available: https://en.wikipedia.org/wiki/COVID-19_pandemic_in_South -Korea

[39] — COVID-19 pandemic in Japan, 2020. [Online]. Available: ] https://en.wikipedia.org/wiki/COVID-19_pandemic_in_Japan

[40] J. Leskovec and A. Krevl, "SNAP Datasets: Stanford large network dataset collection," http://snap.stanford.edu/data Jun. 2014. 